\title{
Parametrices for Symmetric Systems with Multiplicity
}

\author{
Clifford J. Nolan a,*,1, Gunther Uhlmann b,2 \\ ${ }^{a}$ Department of Mathematics and Statistics, University of Limerick, Castletroy, \\ Co. Limerick, Ireland \\ b Department of Mathematics, University of Washington, Seattle, WA 98195, \\ USA
}

\begin{abstract}
We construct microlocal parametrices for generic symmetric systems of partial differential equations having characteristics of variable multiplicity. We apply this to obtain microlocal solutions of Cauchy problems for generic classes of electromagnetic and elasto-dynamical systems.
\end{abstract}

Key words: Anisotropy, parametrix, double characteristics, variable multiplicity

\section{Introduction}

\subsection{Historical background to the problem}

The study of wave propagation in anisotropic media where the wave speeds depend on direction of propagation is very challenging and of great practical importance.

The propagation of singularities and the construction of microlocal parametrices (geometrical optics solutions) for scalar partial differential operators of real principal type like the acoustic wave equation is well understood. By Egorov's theorem, one may conjugate the operator via an invertible Fourier integral

* Corresponding author.

Email address: clifford.nolan@ul.ie (Clifford J. Nolan ).

1 The author acknowledges support from Science Foundation Ireland.

2 The author acknowledges support from NSF and J. S. Gugenheim fellowship. 
operator to the operator $D_{x_{n}}$. The construction of microlocal parametrices for operators of real principal type and the fact that singularities of solutions of these equations propagate along bicharacteristics follow directly from this result $([10])$.

However, most systems of partial differential equations (pde's) in mathematical physics have characteristics with variable multiplicity and do not fit the principal type model. In such instances, striking phenomena occur when the characteristic (wave) speeds coalesce. An example of such a phenomenon is that of conical refraction which consists of the splitting of a light ray at the optical axis of a biaxial crystal into a cone of rays. See [2] for a description of this phenomenon and a historical account. Melrose and Uhlmann [16] constructed a microlocal parametrix for the Cauchy problem for Maxwell's equations in a biaxial crystal with double involutive characteristic sheets. Singularities along the double characteristics (optical axis) propagate along a cone; the cone of conical refraction (see [19]). A more detailed study of the intensity of light at the optical axis was carried out in [21], leading to an explanation of the "double ring phenomenon". The propagation of polarization has been studied in [8]. The propagation of singularities for a class of symmetric systems with double characteristics satisfying a generic condition has been extensively studied in [13] and [14]. The propagation of singularities depends on the behavior of the bicharacteristic flow near the double characteristic variety.

In several physically relevant examples, it is necessary to reconstruct the electromagnetic parameters or elastic parameters of a medium from the trace of the solution of the governing pde on an accessible portion (boundary) of the material. This is an inverse problem. Although propagation of singularities gives some information about the inverse problem, in many cases one needs more quantitative information about the solution. For instance, in [17] the results of [16] and [21] are used to solve an inverse problem for Maxwell's equations in a biaxial crystal when the bicharacteristic sheets are involutive. The results in [17] show how to estimate the singularities (e.g. jump discontinuities) in the electrical permittivity.

A step toward the construction of parametrices for generic symmetric systems was realized in [4]. Braam and Duistermaat consider a system of symmetric pde's, and microlocally conjugate it to normal form, when the system satisfies a generic condition (see more about this below). In related work [5], [6], Yves Colin de Verdiere generalized the latter paper, showing that normal form in the hyperbolic case is actually local. In [5], Colin de Verdiere built an explicit special solution close to the double characteristic variety. Using this, the author is able to relate incoming and outgoing states near the double characteristic variety. Our work differs from the latter in that we build an asymptotic solution (parametrix) for the initial value Cauchy problem, so we are solving a different problem. This paper is an expansion on the preliminary work [18]. 


\subsection{Goals of the paper}

In this paper, we construct a microlocal parametrix for the normal form (hyperbolic case only) developed in [4]. The main goal is to apply the parametrix to microlocally solve the Cauchy problem for a generic class of Maxwell's equations as well as a generic class of equations of elasto-dynamics. The genericity condition implies that the double characteristic variety is non-involutive.

This paper provides a method of geometrical optics for generic anisotropic electromagnetic and elasto-dynamical systems. It is hoped that this work can be built on to provide an efficient description of how electromagnetic and elastic waves scatter from heterogeneities embedded in a generic anisotropic material. Ultimately, we would like to see this work develop into a full-blown inversion method, whereby from measurements of these scattered waves on the surface of a material, one is able to estimate internal heterogeneities (inclusions, interfaces between different materials, etc) contained within the material. Although we do not even begin to approach this inverse problem here, it is our hope that this paper will help to advance this important problem.

\subsection{Outline of paper}

An outline of our construction is as follows. We start be considering systems of pde's with variable multiplicity. Specifically, we consider systems where the principal symbol can vanish to order one (at 'simple characteristic points') or two (at 'double characteristic points') depending on its argument. We construct a transformation that pulls the principal symbol of the original system back to one of principal type. Such a transformation, necessarily cannot be a canonical one (globally) due to the double characteristic points. Therefore, we seek a singular canonical transformation for which an Egorov-type theorem holds, reducing the operator to one with simple characteristics. The solution of the resulting conjugated Cauchy problem proceeds with a singular symbol expansion [1],[11],[12],[16] for the transport coefficients and Hamilton-Jacobi theory for solving the Eikonal equation. The resulting phase is no longer classical but belongs to a singular symbol class itself.

We apply the parametrix construction to microlocally solve the Cauchy problem for the two examples from mathematical physics mentioned above. In doing so, we need to check that the canonical transformations used in [4] to bring the system to normal form can be achieved in such a way that the hypersurface $\{(x, t): t=0\}$ remains space-like. To check this is possible, we need to make use of the structure of the specific pde's. 


\section{Construction of the Parametrix}

\subsection{Preliminaries}

To start, we recall the following theorem ([4], theorem 5), applied to classical pseudodifferential operators.

Theorem 1 Consider the symmetric $q \times q$ linear system $Q u=0$ of classical pseudodifferential operators of order $m$. Suppose that $Q$ has variable multiplicity of at most degree two in a sufficiently small conic microlocal neighborhood $V$ of $\left(0 ; d x_{2}\right) \in \dot{T}^{*}\left(R_{t} \times R_{x}^{3}\right) \equiv T^{*} R_{(t, x)}^{4}-\{0\}$. Under generic restrictions (see [4], Assumption 4 for the precise conditions), $Q$ may be conjugated to normal form as follows. There exists $A: V \rightarrow G L(2, \mathbb{R})$, homogeneous of degree $(1-m) / 2$ such that

$$
\sigma\left(A^{T} Q A\right)=\left[\begin{array}{ccc}
q+r & s & 0 \\
s & \pm(q-r) & 0 \\
0 & 0 & e^{ \pm}
\end{array}\right]
$$

where $\sigma(\cdot)$ denotes the full symbol and $e^{ \pm}$is non-zero in $V$. There exists a Fourier integral operator $\tilde{K}$ of order 0 which is elliptic in $V$ and associated to a canonical transformation $f$ such that $f_{*} \sigma(q)=\tau, f_{*} \sigma(r)=\xi_{1}, f_{*} \sigma(s)=x_{1} \xi_{2}$ modulo an error that is flat at

$$
\Sigma_{2}:=\left\{(t, x, \tau, \xi) \in V: \tau=\xi_{1}=x_{1}=0\right\}
$$

i.e., terms which have their full formal Taylor series vanishing at $\Sigma_{2}$. The Poisson brackets satisfy $\{q, r\}=0,\{q, s\}=0,\{s, r\}=\xi_{2}$ modulo a flat error.

Denoting the left parametrix of $\tilde{K}$ by $\tilde{M}$ in $V$, we then obtain the normal forms of [4]:

$$
M Q K:=\tilde{M} A^{T} Q A \tilde{K} \sim \tilde{P}_{1}^{ \pm}+\tilde{R}_{1}^{ \pm}+\tilde{R}_{0}^{ \pm},
$$

where

$$
\tilde{P}_{1}^{ \pm}:=\left[\begin{array}{ccc}
D_{t}+D_{x_{1}} & x_{1} D_{x_{2}} & 0 \\
x_{1} D_{x_{2}} & \pm\left(D_{t}-D_{x_{1}}\right) & 0 \\
0 & 0 & E_{1}^{ \pm}
\end{array}\right] .
$$


The operators $E_{1}^{ \pm}$are elliptic operators of order 1 and can be solved straightforwardly. Thus, we can restrict our discussion to the upper $2 \times 2$ block system. The symbol of $\tilde{R}_{1}^{ \pm}$is flat at $\Sigma_{2}$ and $\tilde{R}_{0}^{ \pm}$belongs to the class $\Psi^{0}\left(R_{t} \times R_{x}^{n}\right)$ of zero order pseudodifferential operators (in time and space). The symbol $\sim$ means "modulo a smoothing operator" and $\Psi^{m}(X)$ denotes the space of pseudodifferential operators of order $m$ on the open set $X$.

We wish to investigate systems such as Maxwell's equations or those of linear elasticity, where we have well-posedness of the Cauchy problem for the symmetric hyperbolic first order system of pde's

$$
\begin{aligned}
& Q\left(x, D_{t}, D_{x}\right) u(t, x)=0 \\
& \left.u\right|_{t=0}=u_{0} ;\left.u_{t}\right|_{t=0}=u_{1}
\end{aligned}
$$

Multiplying across the negative cofactor matrix of the upper $2 \times 2$ block of (3), yields the Cauchy problem

$$
\begin{gathered}
P^{ \pm} w:=\left( \pm \partial_{t}^{2} \mp \partial_{x_{1}}^{2}-x_{1}^{2} \partial_{x_{2}}^{2}+R_{2}^{ \pm}+P_{1}^{ \pm}+R_{0}^{ \pm}\right) w=0 \\
\left.w\right|_{t=0}=\left.(K u)\right|_{t=0} ;\left.w_{t}\right|_{t=0}=\left.(K u)_{t}\right|_{t=0}
\end{gathered}
$$

where

$$
\begin{gathered}
P_{1}^{ \pm}=A^{ \pm} \partial_{x_{2}}+R_{1}^{ \pm} \\
A^{ \pm}=\left[\begin{array}{cc}
0 & \pm 1 \\
-1 & 0
\end{array}\right]
\end{gathered}
$$

and $R_{2}^{ \pm}, R_{1}^{ \pm}$are second order scalar and first order non-scalar operators respectively, whose symbols are flat and vanish respectively at $\Sigma_{2}$.

We will need to check that the canonical transformation used in [4] to bring the pde (5) to the standard form (3) can be chosen to preserve the space-like set $\{(t, x): t=0\}$, so that in particular, the initial data given in (6) is correct. Also, there is no guarantee that the cofactor system arises from a well posed system of pde's. Therefore, we assume

Assumption 2 The cofactor system of (3) arises from a well posed system of pde and that the canonical transformation $f$ preserves $\{(t, x): t=0\}$.

We verify this assumption in the particular cases of $Q$ to which we apply our method, later on in the applications section. We note that the assumption is 
valid for the formal normal form of [4], since both the normal form its cofactor system are symmetric hyperbolic.

¿From now on, we will consider only the plus superscript case of $P^{ \pm}$. We plan to study the negative case in a follow up paper.

Definition 3 The double characteristic set $\Sigma_{2}$ of $P$ on $V$ is defined by

$$
\begin{aligned}
\Sigma_{2} & :=\{(t, x ; \tau, \xi) \in \operatorname{Char}(\mathrm{P}) \bigcap \mathrm{V}: \operatorname{dp}(\mathrm{t}, \mathrm{x} ; \tau, \xi)=0\} \\
& \equiv\left\{(t, x ; \tau, \xi) \in \operatorname{Char}(\mathrm{P}) \bigcap \mathrm{V}: \tau=\mathrm{x}_{1}=\xi_{1}=0\right\}
\end{aligned}
$$

where

$$
p(t, x ; \tau, \xi)=-\tau^{2}+x_{1}^{2} \xi_{2}^{2}+\xi_{1}^{2}+r_{2}
$$

is the principal symbol of $P$ and $r_{2}$ is flat at $\Sigma_{2}$.

\section{Intertwining to simple characteristic form}

We construct an operator $T$, satisfying $T P \sim B T$ such that $B$ is a pseudodifferential operator with simple characteristics. The operator $T$ is associated to the following singular canonical transformation.

\subsection{Singular canonical transformation}

Define $y^{\prime}=\left(y_{2}, \ldots, y_{n}\right), x^{\prime}=\left(x_{2}, \ldots, x_{n}\right), x^{\prime \prime}=\left(x_{3}, \ldots, x_{n}\right)$, etc. Consider the homogeneous symplectic transformation $\mathcal{T}$ defined by

$$
\begin{aligned}
& x_{1}=\sqrt{2 k / \eta_{2}} \cos \theta \\
& \xi_{1}=-\sqrt{2 k \eta_{2}} \sin \theta \\
& x_{2}=y_{2}+\left(k / 2 \eta_{2}\right) \sin 2 \theta \\
& \xi^{\prime}=\eta^{\prime} \\
& x_{i}^{\prime \prime}=y_{i}^{\prime \prime}
\end{aligned}
$$

where $(x ; \xi),\left(\theta, y^{\prime} ; k, \eta^{\prime}\right)$ are coordinates on the domain $V$ and range of $\mathcal{T}$ respectively. The range is a conic subset of $T^{*}\left(S_{\theta}^{1} \times R_{y^{\prime}}^{n-1}\right)$, where $k \geq 0$ is the 
dual angular $(\theta)$ variable. Observe that this transformation fails to be smooth at the hypersurface $\mathcal{T}\left(\Sigma_{2}\right)=\left\{\left(\theta, y^{\prime}, k=0, \eta^{\prime}\right): \eta_{2} \neq 0\right\}$.

The reason that we consider this kind of transformation is as follows. Intertwining $P$ with a singular integral operator that transforms singularities according to the transformation $\mathcal{T}$ will produce an operator with simple characteristics, as can be seen by a naive application of Egorov's theorem [20] (this is rigorously justified later).

We define a distribution kernel $K_{T}$ of an operator $T$ whose wavefront relation corresponds to $\mathcal{T}$ as follows. Consider the charts $\left\{C_{i}\right\}_{i=1}^{4}$ which cover the circle $S_{\theta}^{1}$ as shown in figure (1). The chart $C_{1}$ contains the north pole, is connected, is symmetric with respect to the diameter joining the north and south poles, and covers slightly less than half of the circle. The other three charts are defined by rotating the first chart respectively by $\pi / 2, \pi, 3 \pi / 2$, so that each pair of these charts has trivial intersection or else has intersection strictly in one of the four quadrants of $S_{\theta}^{1}$.

Fig. 1. Definition of charts $C_{1}, C_{2}, C_{3}, C_{4}$

We will define the 'Hermite' operator $T$ to act as follows

$$
T: \mathcal{E}^{\prime}\left(\mathbb{R}_{t^{\prime}} \times \mathbb{R}_{x}^{n}\right) \rightarrow \mathcal{E}^{\prime}\left(\mathbb{R}_{t} \times S_{\theta}^{1} \times \mathbb{R}_{y^{\prime}}^{n-1} ; L\right)
$$

where the right hand side represents the space of distributional sections of a complex line bundle $L$ over $\mathbb{R}_{t} \times S_{\theta}^{1} \times \mathbb{R}_{y^{\prime}}^{n-1}$ and having transition functions $\exp (i \pi / 4)$. We define $T$ through a pair of operators $T_{1}, T_{2}$ which are in turn defined by the distributional-kernels:

$$
\begin{array}{r}
\left.K_{T_{1}}\left(\left(t^{\prime}, \theta, y^{\prime}\right),(t, x)\right)=\int d \xi e^{i\left\{-\left(x_{1}^{2} \xi_{2} / 2\right) \cot (\theta)+\left(\mathrm{y}^{\prime}-\mathrm{x}^{\prime}\right) \cdot \xi^{\prime}\right.}\right\} \\
\lambda_{1}\left(\theta, y^{\prime}, t, x, \xi\right) \delta\left(t^{\prime}-t\right) \\
\left.K_{T_{2}}\left(\left(t^{\prime}, \theta, y^{\prime}\right),(t, x)\right)=\int d \xi e^{i\left\{\left(\xi_{1}^{2} / 2 \xi_{2}\right) \tan (\theta)+\mathrm{y}^{\prime} \cdot \xi^{\prime}-\mathrm{x} \cdot \xi\right.}\right\} \\
\lambda_{2}\left(\theta, y^{\prime}, t, x, \xi\right) \delta\left(t^{\prime}-t\right)
\end{array}
$$

where $T_{1}$ is defined for $\theta+\pi \in C_{1} \cup C_{3}$ and $T_{2}$ is defined for $\theta+\pi \in C_{2} \cup C_{4}$. One may verify that $K_{T_{1}} \sim K_{T_{2}}$ on overlaps, with an appropriate choice of $\lambda_{1}, \lambda_{2}$. Also, $\lambda_{1}, \lambda_{2}$ are chosen to reflect the fact that whole construction is microlocalized to the conic neighbourhood $V$, and they are chosen to form a partition of unity within $V$. Microlocally, the operators are time independent, and for calculational purposes later, we treat the operator $T$ as being time independent. We define $T$ to be equal to $T_{1}+T_{2}$, where $T_{1}, T_{2}$ are defined by their kernels in (12). 


\subsection{Removal of second order flat terms}

Before proceeding further, we conjugate $P$ by a Fourier integral operator associated to a canonical transformation, so that the second order flat term $r_{2}$ is removed. This can be achieved as follows. The principal symbol $p$ satisfies

$$
\mathcal{T}_{*} p=-\tau^{2}+2 k \eta_{2}+r_{2}
$$

where $r_{2}$ is a flat in the sense that its Taylor series vanishes to all orders at $\mathcal{T}\left(\Sigma_{2}\right)$. The symbol in (13) corresponds to an operator which is of principaltype. As noted in ([15], p.144), if $\tilde{\chi}$ is a canonical transformation from $T^{*}\left(S^{1} \times\right.$ $\left.\mathbb{R}^{n-1}\right)$ to itself that fixes $\mathcal{T}\left(\Sigma_{2}\right)$ to all orders, then it induces a smooth canonical transformation $\chi:=\mathcal{T}^{-1} \tilde{\chi} \mathcal{T}$ from $T^{*} \mathbb{R}^{n}$ to itself.

Definition 4 In view of the previous remarks, we can find an elliptic Fourier integral operator $J$ associated to $\chi$, that removes the second order flat terms, leaves $A \partial_{x_{2}}$ unchanged to first order and maps the operator $R_{1}$ to one whose leading order symbol vanishes on $\Sigma_{2}$.

\subsection{How to deal with odd functions in $\operatorname{Ker}(T)$}

Observe that functions which are odd with respect to $x_{1}$ constitute the kernel $\operatorname{Ker}(T)$ of the operator $T$, and therefore we only expect to be able to invert $T$ on the space of $x_{1}$-even functions. Fortunately, it is possible to represent $x_{1}$ odd functions as even images of the operator $\partial_{x_{1}}-i x_{1} \partial_{x_{2}}$. The latter operator is invertible as the following argument shows. Let

$$
\begin{gathered}
f(x)=\left(\partial_{x_{1}}-i x_{1} \partial_{x_{2}}\right) g(x) \\
f\left(x_{1}, x^{\prime}\right)=-f\left(-x_{1}, x^{\prime}\right) \\
g(x)=g\left(-x_{1}, x^{\prime}\right)
\end{gathered}
$$

The left-parametrix $U$ for the $\left(-\partial_{x_{1}}+i x_{1} \partial_{x_{2}}\right)$ is of the form ([15], Prop. 3.34)

$$
U:=T^{*} N T R
$$

where

$$
\begin{aligned}
& f(x)=x_{1} \tilde{f}(x) \\
& R f(x)=\tilde{f}(x)
\end{aligned}
$$


and $N$ is a pseudodifferential operator.

\subsection{Simplification of lower order terms}

At this stage the operator $P$ has been conjugated to the form

$$
P=L_{2}+A \partial_{x_{2}}+R_{1}+R_{0}
$$

where

$$
A=\left[\begin{array}{cc}
0 & 1 \\
-1 & 0
\end{array}\right], \sigma_{0}\left(R_{1}\right)=i r_{1}:=i\left(r_{1}^{0}+r_{1}^{b}\right)
$$

where

$$
L_{2}:=\partial_{t}^{2}-\partial_{x_{1}}^{2}-x_{1}^{2} \partial_{x_{2}}^{2}
$$

$r_{1}$ is a real-valued matrix symbol that vanishes at $\Sigma_{2}$ and $r_{1}^{b}$ is flat at $\Sigma_{2}$.

We want to intertwine $P$ with $T$ to obtain a simple characteristic operator.

First, however, we will find an elliptic pseudodifferential operator $G \sim \sum_{j=0}^{-\infty} G_{j}$ with $G_{j}$ of order $j$ so that it satisfies the following intertwining property:

$$
G P-\left(L_{2}+E_{1}\left(t, x_{1} D_{x_{1}} D_{x_{2}}^{-1} / 2+x_{2}, x^{\prime \prime}, D_{x^{\prime}}\right)\right) G=0
$$

Remark 5 Note that the argument of $E_{1}, x_{1} D_{x_{1}} D_{x_{2}} / 2+x_{2}=y_{2}$ in the transformed variables $\left(\theta, y, k, \eta^{\prime}\right)$, so that we do not produce symbols involving $\sqrt{k}$, which appears to be the case superficially. Such a symbol would be singular, and as it happens this is just a postponement of having to deal with such singular symbols later.

At the symbol level, equation (20) requires that the first order symbols satisfy

$$
-i\left\{g_{0}, p_{2}\right\}+i g_{0}\left(\xi_{2} A+r_{1}^{0}\right)-e_{1} g_{0}=0
$$

where

$$
p_{2}:=-\tau^{2}+\xi_{1}^{2}+x_{1}^{2} \xi_{2}^{2}
$$


and $e_{1}=e_{1}\left(t, x_{2}+x_{1} \xi_{1} /\left(2 \xi_{2}\right), x^{\prime \prime}, \xi^{\prime}\right)$. Let $C$ be the matrix of normalized eigenvectors of $A$ and $\bar{A}$ be the diagonal matrix of eigenvalues of $A$, i.e.,

$$
C=\frac{1}{\sqrt{2}}\left[\begin{array}{cc}
1 & 1 \\
i & -i
\end{array}\right], \quad \bar{A}=\left[\begin{array}{cc}
i & 0 \\
0 & -i
\end{array}\right]
$$

Making a change of dependent variable from $w$ to $C^{-1} w,(21)$ yields

$$
-\left\{\bar{g}_{0}, p_{2}\right\}+\bar{g}_{0}\left(\xi_{2} \bar{A}+\bar{r}_{1}^{0}\right)+i \bar{e}_{1} \bar{g}_{0}=0
$$

where $\bar{g}_{0}=C^{-1} g_{0} C$, etc.

To satisfy this equation and have $\bar{g}_{0}$ be elliptic near a point of $\Sigma_{2}$, we set

$$
\left.\bar{g}_{0}\right|_{\Sigma_{2}}=\left[\begin{array}{cc}
\alpha_{0} & 0 \\
0 & \beta_{0}
\end{array}\right]
$$

where $\alpha_{0}, \beta_{0}$ are functions of $\left(t, x^{\prime}, \xi^{\prime}\right)$, are non-zero, and these functions will be determined below. The form for $\left.\bar{g}_{0}\right|_{\Sigma_{2}}$ implies that

$$
\bar{e}_{1}=i \xi_{2} \bar{A}
$$

Computing the Poisson bracket in (24) and writing

$$
\bar{g}_{0}=\left[\begin{array}{ll}
a_{1} & b_{1} \\
b_{2} & a_{2}
\end{array}\right], \bar{g}_{0}^{\dagger}=\left[\begin{array}{cc}
0 & -i b_{1} \\
i b_{2} & 0
\end{array}\right]
$$

equation (24) therefore becomes

$$
\left(-\tau \partial_{t}+\xi_{1} \partial_{x_{1}}-\xi_{2}^{2} x_{1} \partial_{\xi_{1}}+x_{1}^{2} \xi_{2} \partial_{x_{2}}\right) \bar{g}_{0}+\xi_{2} \bar{g}_{0}^{\dagger}+\frac{1}{2} \bar{g}_{0} \bar{r}_{1}^{0}=0
$$

We note that for any real-valued matrix symbol $D, \bar{D}$ has the form

$$
\bar{D}=\left[\begin{array}{ll}
\bar{d}_{1} & i \bar{d}_{2} \\
i \bar{d}_{3} & \bar{d}_{4}
\end{array}\right]
$$

where $\bar{d}_{i}, 1 \leq i \leq 4$ are real-valued symbols. 
Consider the space $\mathcal{P}_{j}$ of matrix symbols whose entries are homogeneous of degree $j$ in the variables $\left(t, x_{1}, \tau, \xi_{1}\right)$, with coefficients depending on the parameters $\left(x^{\prime}, \xi^{\prime}\right)$. Introducing symplectic polar coordinates given by (10) with $\xi_{2}=1$, we can identify $\mathcal{P}_{j}$ with the subspace of $C^{\infty}\left(S^{1}, \mathbb{R}^{2 n-1}\right)$ consisting of elements with $C^{\infty}$ extensions to $C^{\infty}\left(\mathbb{R}^{2 n+1}\right)$, homogeneous of degree $j$. We look for a solution of (28) in the form

$$
\left[\begin{array}{ll}
a_{1} & a_{2}
\end{array}\right]^{T} \sim a^{0}+a^{b},\left[\begin{array}{ll}
b_{1} & b_{2}
\end{array}\right]^{T} \sim b^{0}+b^{b}
$$

where $a^{b}, b^{b}$ are flat at $t=x_{1}=\tau=\xi_{1}=0$ and $a^{0}, b^{0}$ are asymptotic sums of formal Taylor series which we determine below in order that (28) is satisfied to all orders near a point $\sigma_{0} \in\left\{(t, x, \tau, \xi) \in \Sigma_{2}: t=0\right\}$. For these Taylor series we write

$$
a^{0} \cong \sum_{j=0}^{\infty} \alpha_{j}^{0}, \quad b^{0} \cong \sum_{j=0}^{\infty} \beta_{j}^{0}
$$

where $A \cong B$ means that $A-B$ vanishes to all orders in the variables $t, x_{1}, \tau, \xi_{1}$ at the point $\sigma_{0}$.

Define the linear operators $\mathcal{T}_{j}, \mathcal{S}_{j}: \mathcal{P}_{j} \rightarrow \mathcal{P}_{j}$ by

$$
\begin{array}{r}
\mathcal{T}_{j}=-\tau \partial_{t}+\xi_{1} \partial_{x_{1}}-x_{1} \partial_{\xi_{1}}+i \bar{A} \\
\mathcal{S}_{j}=-\tau \partial_{t}+\xi_{1} \partial_{x_{1}}-x_{1} \partial_{\xi_{1}}
\end{array}
$$

Projecting (28) into $\mathcal{P}_{j}$, and using (27),(29), we must have

$$
\begin{aligned}
\mathcal{T}_{j} \beta_{j}^{0} & =-x_{1}^{2} \partial_{x_{2}} \beta_{j-2}^{0}+p_{j}^{(1)} \\
\mathcal{S}_{j} \alpha_{j}^{0} & =-x_{1}^{2} \partial_{x_{2}} \alpha_{j-2}^{0}+p_{j}^{(2)}
\end{aligned}
$$

where $p_{j}^{(1)}, p_{j}^{(2)}$ are both real valued linear combinations of previously determined real valued Taylor coefficients $\alpha_{k}^{0}, \beta_{k}^{0}, k<j$. We note that the vector field $x_{1} \partial_{\xi_{1}}-\xi_{1} \partial_{x_{1}}$ appearing in $\mathcal{T}_{j}, \mathcal{S}_{j}$ corresponds to the vector field $\partial_{\theta}$ on $S^{1}$. This observation tells us that $\mathcal{T}_{j}$ is an isomorphism. It also tells us that $\mathcal{S}_{j}$ has a kernel spanned by basis vectors $\tau^{p}\left(x_{1}^{2}+\xi_{1}^{2}\right)^{q} v_{i}$, where $p, q \in \mathbb{N}, p+2 q=$ $j, 1 \leq i \leq 2$, and $v_{1}=\left[\begin{array}{ll}1 & 0\end{array}\right]^{T}, v_{2}=\left[\begin{array}{ll}0 & 1\end{array}\right]^{T}$. The corresponding co-kernel is clearly spanned by basis vectors $t^{p}\left(x_{1}^{2}+\xi_{1}^{2}\right)^{q} v_{i}, p, q \in \mathbb{N}, p+2 q=j, 1 \leq i \leq 2$. We note that the potential cokernel term $t^{j}$ never arises on the right hand side of (33) because (28) is satisfied to all orders of $t$ when $\tau=x_{1}=\xi_{1}=0$. Let us suppose that no co-kernel terms appear on the right hand side of (33) for $j \leq 2 N$, with $N$ a positive integer. If necessary, we can add terms 
$c_{i}\left(x^{\prime}, \xi^{\prime}\right) t^{p}\left(x_{1}^{2}+\xi_{1}^{2}\right)^{q-1} v_{i}, 1 \leq i \leq 2$ to $\alpha_{j-2}^{0}$, and by choosing $\partial_{x_{2}} c_{i}$ appropriately, we can inductively ensure that no cokernel terms arise on the right side of (33) for $j \leq 2(N+1)$. We note that even if $\alpha_{j-2}^{0}$ is zero at $\sigma_{0}$ we can still add the terms $c_{i}\left(x^{\prime}, \xi^{\prime}\right) t^{p}\left(x_{1}^{2}+\xi_{1}^{2}\right)^{q-1} v_{i}$, as long as we ensure that $c_{i}$ vanishes at the values of $x^{\prime}, \xi^{\prime}$ corresponding to $\sigma_{0}$. We can arrange the base case of the induction since we are free to choose $\alpha_{0}=\alpha_{0}^{0}, \beta_{0}=\beta_{0}^{0}$. Finally, since we are dealing with classical pseudodifferential operators, the construction extends by homogeneity to $\xi_{2}>1$.

We have now solved (28) up to a flat error $f_{1}^{b}$. To correct for this error, we write $h_{0}^{b}=\left[\begin{array}{ll}a^{b} & b^{b}\end{array}\right]$, which we require to solve an equation of the form

$$
\left(-\tau \partial_{t}+\xi_{1} \partial_{x_{1}}-\xi_{2}^{2} x_{1} \partial_{\xi_{1}}+x_{1}^{2} \xi_{2} \partial_{x_{2}}\right) h_{0}^{b}+m_{1} h_{0}^{b}=f_{1}^{b}
$$

where $m_{1}$ is $4 \times 4$ matrix symbol of order 1 . In the symplectic polar coordinates, this equation becomes

$$
\left(-\tau \partial_{t}+k \partial_{y_{2}}+\eta_{2} \partial_{\theta}\right) \tilde{h}_{0}^{b}+\tilde{m}_{1} \tilde{h}_{0}^{b}=\tilde{f}_{1}^{b}
$$

where we have arranged for $\tilde{f}_{1}^{b}$ to vanish to all orders at $t=\tau=k=0$. Solving this by the method of variation of parameters, shows that the solution has the same flat property. Transforming back to the original coordinates gives the required smooth correction term $h_{0}^{b}$, which does no affect the ellipticity of $G_{0}$ near $\sigma_{0}$ that we already arranged by the Taylor expansion.

The construction of the lower order symbols is similar but has the following modifications. To satisfy (20) to order $j \leq 0$ we must solve

$$
-i\left\{g_{j-1}, p_{2}\right\}+i \xi_{2}\left[g_{j-1}, A\right]+g_{j-1} r_{1}^{0}-e_{j} g_{0}+q_{j}=0
$$

were $q_{j}$ arise from derivatives of higher order terms previously neglected. Making the change of dependent variable from $w$ to $C^{-1} w$ and substituting our expression for the Poisson bracket, the symbol equation becomes

$$
\begin{array}{r}
\left(-\tau \partial_{t}+\xi_{1} \partial_{x_{1}}-\xi_{2}^{2} x_{1} \partial_{\xi_{1}}+x_{1}^{2} \xi_{2} \partial_{x_{2}}\right) \bar{g}_{j-1} \\
+\xi_{2} \bar{g}_{j-1}^{\dagger}+\bar{g}_{j-1} \bar{r}_{1}^{0}-\bar{e}_{j} \bar{g}_{0}+\bar{q}_{j}=0
\end{array}
$$

We choose $\left.\bar{g}_{j-1}\right|_{\sigma_{0}}$ to be of the same form as (25) but where $\alpha_{0}, \beta_{0}$ are now of degree $j-1$. Then setting $\tau=x_{1}=\xi_{1}=0$, we see that $\bar{e}_{j}$ is determined by

$$
\bar{e}_{j}\left(t, x^{\prime}, \xi^{\prime}\right)=\left.\bar{q}_{j} \bar{g}_{0}^{-1}\right|_{\tau=x_{1}=\xi_{1}=0}
$$


We can again construct a Taylor series in a similar fashion as we did for $\bar{g}_{0}$ to solve (37) for $\bar{g}_{j-1}$ up to a flat error. We add a flat correction term $\bar{g}_{j-1}^{b}$ to get (37) holding exactly. This is the same as we did for the order one symbol equation, except now there are extra flat terms, arising from $\bar{q}_{j}-\bar{e}_{j} \bar{g}_{0}$, which we must also correct for too.

\subsection{Solution Operator for the Cauchy Problem}

The results of the previous section have shown us that in order to solve the Cauchy problem

$$
\begin{gathered}
\left(L_{2}+R_{2}+R_{1}+R_{0}\right) u=0 \\
\left.u\right|_{t=0}=u_{0} \\
\left.u_{t}\right|_{t=0}=0
\end{gathered}
$$

it is enough to solve

$$
\begin{gathered}
\left(L_{2}+A \partial_{x_{2}}+E_{0}\right) v=0 \\
\left.v\right|_{t=0}=G u_{0} \\
\left.v_{t}\right|_{t=0}=0
\end{gathered}
$$

where $v=G u$ and $E_{0}$ has the same special form as $E_{1}$ in the previous section, but is of order zero.

We will simplify (40) further by considering the corresponding Cauchy problem satisfied by $T v$ but we have to take care of the the $x_{1}$-odd functions in the kernel of $T$ first. If $v\left(x_{1}, x^{\prime}\right)=-v\left(-x_{1}, x^{\prime}\right)$ then the results of the second last section show that we can write

$$
v=\left(-\partial_{x_{1}}+i x_{1} \partial_{x_{2}}\right) \tilde{v}
$$

so that $\tilde{v}=U v$ in view of (15). The corresponding Cauchy problem to be satisfied by $\tilde{v}$ is of the form

$$
\begin{gathered}
\left(L_{2}+(A-2 i I) \partial_{x_{2}}+E_{0}\right) \tilde{v}=0 \\
\left.\tilde{v}\right|_{t=0}=U G u_{0} \\
\left.\tilde{v}_{t}\right|_{t=0}=0
\end{gathered}
$$


The adjustment to the first order differential operator arises in (42) from the commutator of $\partial_{x_{1}}-i x_{1} \partial_{x_{2}}$ with $L_{2}$.

The following notation will be convenient

Definition 6 We can decompose a distribution u into its odd and even components using the notation:

$$
\begin{gathered}
u=u^{\text {odd }}+u^{\text {even }} \\
u^{\text {even }}=\pi_{\text {even }} u(x):=\left(u\left(x_{1}, x^{\prime}\right)+u\left(-x_{1}, x^{\prime}\right)\right) / 2 \\
u^{\text {odd }}=\pi_{\text {odd }} u(x):=\left(u\left(x_{1}, x^{\prime}\right)-u\left(-x_{1}, x^{\prime}\right)\right) / 2
\end{gathered}
$$

Remark 7 It now follows that one can solve the original Cauchy problem (39) by making the decomposition (43) and solving the Cauchy problems $(40,42)$. Our remaining task is to show how to solve these initial value problems.

\subsection{Egorov-type reduction}

Although Egorov's theorem does not strictly apply, one can verify in analogous manner to the proof of the Egorov theorem (see the intertwining derivation in p.147 of [15] for more details)

$$
L_{2} T^{*}=T^{*}\left(\partial_{t}^{2}-2 \partial_{\theta} \partial_{y_{2}}\right)
$$

Our task is now reduced to solving the following Cauchy problem

$$
\begin{gathered}
B w:=\left(-\partial_{t}^{2}+\partial_{\theta} \partial_{y_{2}}-A^{\prime} \partial_{y_{2}}-B_{0}\left(t, y^{\prime}, D_{y^{\prime}}\right)\right) w=0 \\
\left.w\right|_{t=0}=w_{0} \\
\left.w_{t}\right|_{t=0}=0
\end{gathered}
$$

where we assume without loss of generality that the matrix $-A^{\prime}$ has been diagonalized with purely imaginary eigenvalues $i,-i$ for even data and $3 i, i$ for odd data and we have also rescaled $\theta$ for convenience.

Also, our odd / even decomposition allows us to assume without loss of generality that $w_{0}$ has non-negative angular frequencies $(k \geq 0)$. Indeed, (45) is 
to be solved in $\mathcal{T}(V)$ where

$$
0 \leq k \leq \epsilon \eta_{2}
$$

with $0<\epsilon$, a constant depending on the size of $V$.

\subsection{Solution operator}

We seek a solution operator $w_{0} \mapsto w$ in the form of a Fourier-Integral Series pair [15]:

$$
\begin{aligned}
& w=\sum_{ \pm, k \geq 0} \int\left[\begin{array}{cc}
e^{i \phi_{ \pm}^{(1)}\left(t, k, \eta^{\prime}\right)} & 0 \\
0 & e^{i \phi_{ \pm}^{(2)}\left(t, k, \eta^{\prime}\right)}
\end{array}\right] \\
& (2 \pi)^{-n} e^{i\left((4 k+1)(\theta-\tilde{\theta})+\left(y^{\prime}-\tilde{y}^{\prime}\right) \cdot \eta^{\prime}\right)} e_{ \pm}\left(t, y^{\prime}, k, \eta^{\prime}\right) w_{0}\left(\tilde{\theta}, \tilde{y}^{\prime}\right) d \tilde{\theta} d \tilde{y}^{\prime} d \eta^{\prime}
\end{aligned}
$$

where $\phi_{ \pm}^{(j)}$ are phase functions to be determined and $e_{ \pm}$is a $2 \times 2$ matrix of symbols also to be determined.

Remark 8 Notice that we have only taken positive frequencies as the data is in the image of $T$ and we have only taken every other odd frequency due to the fact that after rescaling $\theta$, images of $T$ are odd under a shift by a quarter cycle.

Application of the operator $B$, defined in (45) yields

$$
\begin{aligned}
& B w=\sum_{ \pm, k \geq 0} \int\left[\begin{array}{cc}
e^{i \phi_{ \pm}^{(1)}\left(t, k, \eta^{\prime}\right)} & 0 \\
0 & e^{i \phi_{ \pm}^{(2)}\left(t, k, \eta^{\prime}\right)}
\end{array}\right] \\
& (2 \pi)^{-n} e^{i\left((4 k+1)(\theta-\tilde{\theta})+\left(y^{\prime}-\tilde{y}^{\prime}\right) \cdot \eta^{\prime}\right)} r_{ \pm} e_{ \pm}\left(t, y^{\prime}, k, \eta^{\prime}\right) w_{0}\left(\tilde{\theta}, \tilde{y}^{\prime}\right) d \tilde{\theta} d \tilde{y}^{\prime} d \eta^{\prime}
\end{aligned}
$$

with

$$
\begin{array}{r}
r_{ \pm}=\left[\begin{array}{cc}
\left(\partial_{t} \phi_{ \pm}^{(1)}\right)^{2}-\left(4 k+1+\nu_{1}\right) \eta_{2} & 0 \\
0 & \left(\partial_{t} \phi_{ \pm}^{(2)}\right)^{2}-\left(4 k+1+\nu_{2}\right) \eta_{2}
\end{array}\right]+ \\
i\left[\begin{array}{rr}
-2 \partial_{t} \phi_{ \pm}^{(1)} \partial_{t}+\left(4 k+1+\nu_{1}\right) \partial_{y_{2}} & 0 \\
0 & -2 \partial_{t} \phi_{ \pm}^{(2)} \partial_{t}+\left(4 k+1+\nu_{2}\right) \partial_{y_{2}}
\end{array}\right]+ \\
\left\{\partial_{t}^{2}-r_{0}^{ \pm}\right\}
\end{array}
$$


where the possible values of $\left(\nu_{1}, \nu_{2}\right)$ are $(1,-1)$ for even data and $(3,1)$ for odd data respectively. Note that $r_{0}^{ \pm}$maps $S^{\left(m_{1}, m_{2}\right)} \rightarrow S^{\left(m_{1}, m_{2}\right)}$, where $S^{\left(m_{1}, m_{2}\right)}$ denotes the class of symbol valued symbols [16] in the variables $k, \eta^{\prime}$.

To satisfy (45) to leading order of singularity, it is necessary to have

$$
\phi_{ \pm}^{(j)}= \pm t \sqrt{\left(4 k+1+\nu_{j}\right) \eta_{2}}, \quad j=1,2
$$

We observe that these phase functions belong to the symbol-valued symbol class $S^{(1 / 2,1 / 2)}$ with respect to the frequencies $\left(k, \eta^{\prime}\right)$.

We write

$$
e_{ \pm}=\left[\begin{array}{c}
e_{ \pm}^{(1)} \\
e_{ \pm}^{(2)}
\end{array}\right]
$$

where $e_{ \pm}^{(j)}, j=1,2$ are $1 \times 2$ row vectors of symbols.

To satisfy (45) to the next order of singularity, we must solve the following transport equations $M_{ \pm} e_{ \pm}=0$ where

$$
\begin{array}{r}
M_{ \pm}=\left[\begin{array}{cc}
\sqrt{\left(4 k+1+\nu_{1}\right) \eta_{2}} & 0 \\
0 & \sqrt{\left(4 k+1+\nu_{2}\right) \eta_{2}}
\end{array}\right] \partial_{t} \\
\mp \frac{1}{2}\left[\begin{array}{cc}
4 k+1+\nu_{1} & 0 \\
0 & 4 k+1+\nu_{2}
\end{array}\right] \partial_{y_{2}} \\
\\
\\
\end{array}
$$

with initial conditions

$$
\begin{aligned}
&\left.\left(e_{+}+e_{-}\right)\right|_{t=0} \sim I \\
& {\left[\begin{array}{cc}
\sqrt{\left(4 k+1+\nu_{1}\right) \eta_{2}} & 0 \\
0 & \sqrt{\left(4 k+1+\nu_{2}\right) \eta_{2}}
\end{array}\right] \begin{array}{l}
\left.\left(e_{+}-e_{-}\right)\right|_{t=0} \sim \\
\left.i \partial_{t}\left(e_{+}+e_{-}\right)\right|_{t=0}
\end{array} }
\end{aligned}
$$

For odd data, can rewrite $(52)$ as $M_{ \pm}^{\prime} e_{ \pm}=0$ with 


$$
M_{ \pm}^{\prime}=\partial_{t} \mp\left[\begin{array}{cc}
\alpha_{1} & 0 \\
0 & \alpha_{2}
\end{array}\right] \quad \partial_{y_{2}} \mp C^{ \pm}
$$

where

$$
\begin{array}{r}
\alpha_{j}=\frac{1}{2} \sqrt{\left(4 k+1+\nu_{j}\right) / \eta_{2}}, j=1,2 \\
C^{ \pm}=\frac{i}{2}\left[\begin{array}{cc}
\frac{1}{\sqrt{\left(4 k+1+\nu_{1}\right) \eta_{2}}} & 0 \\
0 & \frac{1}{\sqrt{\left(4 k+1+\nu_{1}\right) \eta_{2}}}
\end{array}\right]\left(\partial_{t}^{2}-r_{0}^{ \pm}\right)
\end{array}
$$

belong to the symbol class $S^{(0,0)}$ because $k / \eta_{2} \leq \epsilon$. We can rewrite the transport equations as ode system

$$
\left(\frac{d}{d t} \pm C^{ \pm}\right)\left[\begin{array}{l}
e_{ \pm}^{(1)}\left(t, y_{2} \mp \alpha_{1} t, y^{\prime \prime}, k, \eta^{\prime}\right) \\
e_{ \pm}^{(2)}\left(t, y_{2} \mp \alpha_{2} t, y^{\prime \prime}, k, \eta^{\prime}\right)
\end{array}\right]=0
$$

As in regular geometrical optics, we then solve a hierarchy of such (possibly inhomogeneous) transport equations with

$$
e_{ \pm} \sim \sum_{m=0}^{\infty} e_{ \pm, m}
$$

and

$$
e_{ \pm} \in S^{0,0} ; \quad e_{ \pm, m} \in S^{-m / 2,-m / 2}
$$

We set $e_{ \pm, 0}=\frac{1}{2} I$ and $e_{-, m}=-e_{+, m}$ for $m \geq 1$ at $t=0$. Then the solution to the transport equations allows us to calculate $\partial_{t} e_{ \pm, m}$ at $t=0$ in terms of $\mathrm{e}_{ \pm, m}$ at $t=0$. Substituting these values in (53) recursively gives initial conditions for $e_{+, m}, m \geq 1$ in order to satisfy (53).

For even data with $k \geq 1$, we can solve the transport equations in exactly the same way as we did for the odd data.

Therefore, we are left just to solve the transport equations for even data with $k=0$, in which case $\alpha_{2}=0, \nu_{1}=1$. This effectively replaces (54) by

$$
M_{ \pm}^{\prime}=\left[\begin{array}{ll}
\partial_{t} & 0 \\
0 & 0
\end{array}\right] \mp\left[\begin{array}{cc}
\alpha_{1} & 0 \\
0 & 0
\end{array}\right] \partial_{y_{2}} \mp C^{ \pm}
$$


where

$$
C^{ \pm}=i \frac{\partial_{t}^{2}-r_{0}^{ \pm}}{\sqrt{2 \eta_{2}}} ; \quad \alpha_{1}=\frac{1}{\sqrt{2 \eta_{2}}}
$$

Writing the zero order matrix symbol operator $r_{0}^{ \pm}$as

$$
r_{0}^{ \pm}=-\left[\begin{array}{ll}
r_{11}^{ \pm} & r_{12}^{ \pm} \\
r_{21}^{ \pm} & r_{22}^{ \pm}
\end{array}\right]
$$

we see that $M_{ \pm}^{\prime} e_{ \pm}=0$ gives the following two equations to be solved

$$
\begin{aligned}
& \left(\partial_{t} \mp \frac{1}{\sqrt{2 \eta_{2}}} \partial_{y_{2}}\right) e_{ \pm}^{(1)} \mp i \frac{\partial_{t}^{2} e_{ \pm}^{(1)}}{2 \sqrt{2 \eta_{2}}} \mp i \frac{r_{11}^{ \pm} e_{ \pm}^{(1)}+r_{12}^{ \pm} e_{ \pm}^{(2)}}{2 \sqrt{2 \eta_{2}}}=0 \\
& \partial_{t}^{2} e_{ \pm}^{(2)} \mp r_{21}^{ \pm} e_{ \pm}^{(1)} \mp r_{22}^{ \pm} e_{ \pm}^{(2)}=0
\end{aligned}
$$

We will construct a pair $e_{ \pm}^{(j)}, j=1,2$ with

$$
e_{ \pm}^{(j)} \sim \sum_{m=0}^{\infty} e_{ \pm, m}^{(j)}, j=1,2
$$

and

$$
e_{ \pm}^{(j)} \in S^{0} ; \quad e_{ \pm, m}^{(j)} \in S^{-m / 2}
$$

Indeed, we can solve (62) for $e_{ \pm, m}^{(1)}$ in advance of solving (63), due to the disparity of homogeneity in $\eta_{2}$ factors of $e_{ \pm}^{(1)}$ and $e_{ \pm}^{(2)}$ in equation (62). All coefficients except that of the $\partial_{t}$ are in $S^{-1 / 2}$. The value of $e_{ \pm, m}^{(1)}$ can then be used in (63) to solve for $e_{ \pm, m}^{(2)}$

The initial conditions that need to be satisfied are now modified to

$$
\begin{aligned}
& e_{+}^{(1)}+e_{-}^{(1)}=\left[\begin{array}{ll}
1 & 0
\end{array}\right] \\
&\left.\left.\sqrt{2 \eta_{2}}\left(e_{+}^{(1)}-e_{-}^{(1)}\right)\right|_{t=0} \sim i \partial_{t}\left(e_{+}^{(1)}+e_{-}^{(1)}\right)\right|_{t=0} \\
& e_{+}^{(2)}+e_{-}^{(2)}=\left[\begin{array}{ll}
0 & 1
\end{array}\right] \\
&\left.\partial_{t}\left(e_{+}^{(2)}+e_{-}^{(2)}\right)\right|_{t=0}=\left[\begin{array}{ll}
0 & 0
\end{array}\right]
\end{aligned}
$$


As before, we can recursively derive initial conditions for $e_{ \pm, m}^{(1)}$ to satisfy the first two initial conditions in (66). We set $\left.e_{ \pm, 0}^{(2)}\right|_{t=0}=\left[\begin{array}{ll}0 & \frac{1}{2}\end{array}\right]$ and $\left.e_{ \pm, m}^{(2)}\right|_{t=0}=$ $\left.\partial_{t} e_{ \pm, m}^{(2)}\right|_{t=0}=\left[\begin{array}{ll}0 & 0\end{array}\right], m \geq 1$, to satisfy the second two initial conditions in (66).

Remark 9 The $k=0$ Fourier coefficient term does not propagate singularities within the double characteristic variety. The underlying reason for this is due to the fact that time-derivative coefficient in the transport operator $M_{ \pm}$ contains a factor $\sqrt{k \eta_{2}}$ (for large $k$ ), whereas in [16], the coefficient contained a factor analogous to $k$ which necessitated a conical refraction correction term that led to propagation of singularities within the double characteristic variety. The $k>0$ terms propagate singularities along characteristics of the principal type operator $B$.

We have just seen how to solve

$$
\begin{gathered}
B w=0 \\
\left.w\right|_{t=0}=w_{0} ;\left.w_{t}\right|_{t=0}=0
\end{gathered}
$$

Using similar arguments and superposition, we can build an explicit microlocal solution to the Cauchy problem

$$
\begin{gathered}
B w=0 \\
\left.w\right|_{t=0}=w_{0} ;\left.w_{t}\right|_{t=0}=w_{1}
\end{gathered}
$$

and we denote the solution operator of (68) by $S(t)$.

\subsection{Completing the parametrix construction}

We are almost in a position to put all the pieces together, and write down a solution operator for the original Cauchy problem (5) in the $t, x$ variables. The last result we will need is

Lemma 10 [15] The operator $T T^{*}$ is a singular pseudodifferential operator in the sense

$$
T T^{*}=\tilde{L}\left(y^{\prime}, D_{y^{\prime}}, D_{\theta}\right) \alpha\left(y^{\prime}, D_{y^{\prime}}\right) \beta\left(D_{\theta}\right)
$$

where $\alpha, \beta$ are pseudodifferential operators in the variables of their arguments - their product is not a pseudodifferential operator in their joint variables. Also, $\tilde{L}$ is a zero order, microlocally elliptic, pseudodifferential operator. 
Proof: It suffices to calculate the contribution to the kernel $K_{T T^{*}}^{(1,3)}$ of $T T^{*}$ from charts $C_{1}, C_{3}$; the contribution from the other pairs of charts being similar. Suppressing the trivial time dependence of the kernels, we have

$$
\begin{gathered}
K_{T T^{*}}^{(1,3)}\left(y^{\prime}, \theta, \bar{y}^{\prime}, \sigma\right)=\int \exp \left(i \Phi\left(y^{\prime}, \theta, \bar{y}^{\prime}, \sigma, \eta, \xi\right)\right) a\left(\theta, \sigma, \xi_{2}, \eta_{2}\right) d x d \xi d \eta \\
\Phi=x \cdot(\xi-\eta)+y^{\prime} \cdot \eta^{\prime}-\bar{y}^{\prime} \cdot \xi^{\prime}+\left(\eta_{1}^{2} / 2 \eta_{2}\right) \tan (\theta)-\left(\xi_{1}^{2} / 2 \xi_{2}\right) \tan (\sigma)
\end{gathered}
$$

Here $a\left(\theta, \sigma, \xi_{2}, \eta_{2}\right)$ incorporates the products of the $\lambda_{1}, \lambda_{2}$ symbols. Performing a stationary phase calculation in the $(x, \xi)$ variables, we reduce to

$$
\begin{gathered}
K_{T T^{*}}^{(1,3)}\left(y^{\prime}, \theta, \bar{y}^{\prime}, \sigma\right)=\int \exp \left(i \Phi\left(y^{\prime}, \theta, \bar{y}^{\prime}, \sigma, \eta\right)\right) A(\theta, \sigma) \eta_{2} d \eta \\
\Phi=\left(y^{\prime}-\bar{y}^{\prime}\right) \cdot \eta^{\prime}+\left(\eta_{1}^{2} / 2 \eta_{2}\right)(\tan (\theta)-\tan (\sigma))
\end{gathered}
$$

Making a Taylor expansion, we write

$$
\tan \theta-\tan \sigma=h(\theta, \sigma)(\theta-\sigma)
$$

where $h$ does not vanish in $C_{1} \cup C_{3}$. Making a change of variables

$$
\bar{\eta}_{1}=\left(\eta_{1}^{2} / 2 \eta_{2}\right) h(\theta, \sigma)
$$

we obtain

$$
\begin{gathered}
K_{T T^{*}}^{(1,3)}\left(y^{\prime}, \theta, \bar{y}^{\prime}, \sigma\right)=\int \exp \left(i\left(\left(y^{\prime}-\bar{y}^{\prime}\right) \cdot \eta^{\prime}+\bar{\eta}_{1}(\theta-\sigma)\right)\right) \\
\eta_{2}^{3 / 2} \frac{A(\theta, \sigma)}{\sqrt{h(\theta, \sigma) \bar{\eta}_{1}}} d \eta^{\prime} d \bar{\eta}_{1}
\end{gathered}
$$

The ability of the amplitude of the latter oscillatory integral to blow up at $\bar{\eta}_{1}=$ 0 demonstrates the singular symbol associated to singular Pseudodifferential operators. Identifying

$$
\begin{array}{r}
K_{\alpha}\left(y^{\prime}, \bar{y}^{\prime}\right)=\int \exp \left(i\left(y^{\prime}-\bar{y}^{\prime}\right) \cdot \eta^{\prime}\right) \eta_{2}^{3 / 2} d \eta^{\prime} \\
K_{\beta}(\theta, \sigma)=\int \exp \left(i(\theta-\sigma) \bar{\eta}_{1}\right) \frac{A(\theta, \sigma)}{\sqrt{h(\theta, \sigma) \bar{\eta}_{1}}} d \bar{\eta}_{1}
\end{array}
$$

and letting $\tilde{L}$ represent the effect of microlocalizing the operator $T T^{*}$ to the appropriate chart, the lemma is proved. 
Corollary 11 The operator $T T^{*}$ has a left microlocal parametrix $Y$ :

$$
Y=m\left(y^{\prime}, D_{y^{\prime}}, D_{\theta}\right) \alpha^{-1} \beta^{-1}
$$

where $m$ is a (microlocal) parametrix for $\tilde{L}$.

Collecting the above results we obtain the final form for the solution operator for the Cauchy problem (39).

Theorem 12 The operator $\mathcal{E}$ given by

$$
\begin{gathered}
\mathcal{E}=M J^{-1} G^{-1} T^{*} Y S T G \pi_{\text {even }} J K+ \\
M J^{-1} G^{-1}\left(D_{x_{1}}-i x_{1} D_{x_{2}}\right) T^{*} Y S T R G \pi_{\text {odd }} J K
\end{gathered}
$$

is a solution operator for the Cauchy problem (39). Here, $M, J, L$ are microlocally elliptic Fourier integral operators associated to canonical transformations. The operator $Y$ is a product of pseudodifferential operators in the $\theta$ and $y_{2}$ variables respectively. $G$ is a pseudodifferential operator. $\pi_{\text {odd }}, \pi_{\text {even }}$ project data to the odd and even components with respect to the involution $x_{1} \mapsto-x_{1}$. $T$ is a Fourier integral operator associated to a singular canonical transformation. The operator $S$ is the solution operator for the Cauchy problem associated to the simple characteristic operator $B$.

\section{Application to Electromagnetism and Linear Elasticity}

In this section, we examine the specific pde systems governing electromagnetism and linear elasticity. In solving the Cauchy problem associated to (4), we are implicitly assuming that the canonical transformation $f$ in [4] applied to bring $Q$ to the normal form can be chosen so that it preserves the spacelikeness of $\{(t, x): t=0\}$ and so we still have to check that this can be arranged in the cases where $Q$ is associated to electromagnetism and linear elasticity. We will use the notation of [4], which differs from our earlier notation.

Specifically, we examine the construction in (Proposition 6, [4]). In the proof of this proposition, one initially writes the symbol $\sigma(Q)$ of $Q$ (in a suitable basis) using independent functions $q, r, s$ (whose joint zero-set defines $\Sigma_{2}$ )

$$
\sigma(Q)=\left[\begin{array}{cc}
q+r & s \\
s & q-r
\end{array}\right]
$$


The next step is to conjugate $\sigma(Q)$ by a non-singular $A$ with entries

$$
A:=\left[\begin{array}{ll}
a & b \\
c & d
\end{array}\right]
$$

to get

$$
A \sigma(Q) A^{T}=\left[\begin{array}{cc}
\tilde{q}+\tilde{r} & \tilde{s} \\
\tilde{s} & \tilde{q}-\tilde{r}
\end{array}\right]
$$

where

$$
\begin{aligned}
& \tilde{q}=q\left(a^{2}+b^{2}+c^{2}+d^{2}\right) / 2+r\left(a^{2}-b^{2}+c^{2}-d^{2}\right) / 2+s(a b+c d) \\
& \tilde{r}=q\left(a^{2}+b^{2}-c^{2}-d^{2}\right) / 2+r\left(a^{2}-b^{2}-c^{2}+d^{2}\right) / 2+s(a b-c d) \\
& \tilde{s}=q(a c+b d)+r(a c-b d)+s(a d+b c)
\end{aligned}
$$

The canonical transformation $f$ is constructed in three possible cases. We use $(t, x ; \tau, \xi)$ for the original coordinates and $(\bar{t}, \bar{x} ; \bar{\tau}, \bar{\xi})$ for the new coordinates after transforming by $f$.

In the first case (this is the $(++)$ case in the notation of [4], page 9), the transformation $f$ is given by

$$
\bar{\tau}=\lambda \tilde{q}, \bar{\xi}_{1}=\lambda \tilde{r}, \bar{x}_{2}=\tilde{s} / \lambda, \bar{\xi}_{2}=\lambda^{2}
$$

where $\lambda$ is a positive function and the transformation is completed via Darboux's construction [9]. We need to check that

$$
\left.\frac{\partial \bar{\tau}}{\partial \tau}\right|_{\Sigma_{2}} \neq 0
$$

in order to solve the initial value problem

$$
\begin{aligned}
\{\bar{\tau}, \bar{t}\} & =1 \\
\left.\bar{t}\right|_{t=0} & =0
\end{aligned}
$$

in the Darboux construction.

We need to use the properties of the specific operator $Q$ in order to check the condition (83). First we consider the case of the electromagnetic field in a 
biaxial crystal. The governing equation for the electric field $E$ associated with a permittivity tensor $\epsilon$ is

$$
\left(\epsilon(x) \partial_{t}^{2}+\nabla \times \nabla \times\right) E(x, t)=0 .
$$

Assuming we are dealing with a crystal optics, $\epsilon$ has the diagonal form

$$
\epsilon(x)=\left[\begin{array}{ccc}
\epsilon_{1}(x) & 0 & 0 \\
0 & \epsilon_{2}(x) & 0 \\
0 & 0 & \epsilon_{3}(x)
\end{array}\right]
$$

with the generic condition $0<\epsilon_{1}(x)<\epsilon_{2}(x)<\epsilon_{3}(x)$, the symbol of (85) is

$$
\left[\begin{array}{ccc}
\epsilon_{1} \tau^{2}-\xi_{2}^{2}-\xi_{3}^{2} & \xi_{1} \xi_{2} & \xi_{1} \xi_{3} \\
\xi_{1} \xi_{2} & \epsilon_{2} \tau^{2}-\xi_{1}^{2}-\xi_{3}^{2} & \xi_{2} \xi_{3} \\
\xi_{1} \xi_{3} & \xi_{2} \xi_{3} & \epsilon_{3} \tau^{2}-\xi_{1}^{2}-\xi_{2}^{2}
\end{array}\right]
$$

We write the symbol (87) in the block form

$$
\left[\begin{array}{ll}
Q_{L L} & Q_{L R} \\
Q_{L R}^{T} & Q_{R R}
\end{array}\right]
$$

where $Q_{R R}$ is an elliptic pseudodifferential operator. We can do this in a suitable basis because the diagonal elements in (87) don't simultaneously vanish at characteristic points (corresponding to the fact that the inner characteristic sheet of the characteristic variety of $Q$ is always separated from the outer two - see [7], p. 604). The second diagonal element actually vanishes at $\Sigma_{2}$ (see [4]) and so we deduce that $Q_{L L}$ can be written in one of the following two forms

$$
\begin{aligned}
& Q_{L L}^{(i)}=\left[\begin{array}{cc}
\epsilon_{1} \tau^{2}-\xi_{2}^{2}-\xi_{3}^{2} & \xi_{1} \xi_{2} \\
\xi_{1} \xi_{2} & \epsilon_{2} \tau^{2}-\xi_{1}^{2}-\xi_{3}^{2}
\end{array}\right] \\
& Q_{L L}^{(i i)}=\left[\begin{array}{cc}
\epsilon_{3} \tau^{2}-\xi_{1}^{2}-\xi_{2}^{2} & \xi_{2} \xi_{3} \\
\xi_{2} \xi_{3} & \epsilon_{2} \tau^{2}-\xi_{1}^{2}-\xi_{3}^{2}
\end{array}\right]
\end{aligned}
$$

We will work out what happens for (89), noting that similar arguments apply for $(90)$. 
We will use the same notation as in ([4], Lemma 1). This means that the operator $P$ defined below now has a different meaning from that used earlier in this paper. Thus, as in $([4])$, we have

$$
P=Q_{L L}-Q_{L R} Q_{R R}^{-1} Q_{R L}
$$

With the definitions

$$
g=\xi_{1} \xi_{3} ; h=\xi_{2} \xi_{3} ; j=\left(\epsilon_{3} \tau^{2}-\xi_{1}^{2}-\xi_{2}^{2}\right)^{-1}
$$

we have

$$
P=\left[\begin{array}{cc}
\epsilon_{1} \tau^{2}-\xi_{2}^{2}-\xi_{3}^{2} & \xi_{1} \xi_{2} \\
\xi_{1} \xi_{2} & \epsilon_{2} \tau^{2}-\xi_{1}^{2}-\xi_{3}^{2}
\end{array}\right]-j\left[\begin{array}{cc}
g^{2} & g h \\
g h & h^{2}
\end{array}\right]
$$

We have to check

$$
\frac{\partial}{\partial \tau}\left(q\left(a^{2}+b^{2}+c^{2}+d^{2}\right) / 2+r\left(a^{2}-b^{2}+c^{2}-d^{2}\right) / 2+s(a b+c d)\right) \neq 0(94)
$$

on $\Sigma_{2}$. By inspection of (93),

$$
\begin{array}{r}
q=\left(\epsilon_{1} \tau^{2}+\epsilon_{2} \tau^{2}-\xi_{1}^{2}-\xi_{2}^{2}-2 \xi_{3}^{2}\right) / 2-j\left(g^{2}+h^{2}\right) / 2 \\
r=\left(\epsilon_{1} \tau^{2}-\epsilon_{2} \tau^{2}+\xi_{1}^{2}-\xi_{2}^{2}\right) / 2-j\left(g^{2}-h^{2}\right) / 2 \\
s=\xi_{1} \xi_{2}-j g h
\end{array}
$$

Recalling that $q, r, \xi_{2}$ (see [4]) vanish at $\Sigma_{2}$, we also have $s, h$ vanishing there. Consequently, (94) is equivalent to

$$
\tau \epsilon_{1}\left(a^{2}+c^{2}\right)+\tau \epsilon_{2}\left(b^{2}+d^{2}\right)+2 \tau \epsilon_{3} j^{2} g^{2}\left(a^{2}+c^{2}\right) \neq 0
$$

This is clearly true since we can assume $|\tau| \gg 1$ and $A$ is non-singular. Warning: do not confuse this $\tau$ with the $\tau$ in the normal form from [4], which is allowed to be zero!

For linear elasticity, the governing equation for the displacement vector field $u$ associated to the Hooke's tensor $c_{i j k l}$ and density field $\rho$ is

$$
\frac{\partial^{2} u_{i}}{\partial t^{2}}-\frac{1}{\rho} \frac{\partial\left(c_{i j k l} \frac{\partial u_{k}}{\partial x_{l}}\right)}{\partial x_{j}}=0
$$


and hence the associated leading order symbol is

$$
Q_{i j}=-\rho \tau^{2} \delta_{i j}+c_{i j k l} \xi_{j} \xi_{l}
$$

In an elastic material with cubic symmetry, the symbol $Q$ is [3]

$$
-\rho \tau^{2}+\left[\begin{array}{ccc}
A \xi_{1}^{2}+L\left(\xi_{2}^{2}+\xi_{3}^{2}\right) & \mu \xi_{1} \xi_{2} & \mu \xi_{1} \xi_{3} \\
\mu \xi_{1} \xi_{2} & A \xi_{2}^{2}+L\left(\xi_{1}^{2}+\xi_{3}^{2}\right) & \mu \xi_{2} \xi_{3} \\
\mu \xi_{1} \xi_{3} & \mu \xi_{2} \xi_{3} & A \xi_{3}^{2}+L\left(\xi_{1}^{2}+\xi_{2}^{2}\right)
\end{array}\right]
$$

where $A, F, L$ are the three elastic coefficients characterizing the material, and $\mu=F+L$. The eight double characteristic points are $\xi=\tau \sqrt{\rho /(A+L-F)}( \pm 1, \pm 1, \pm 1)$ where the upper $2 \times 2$ block has non-trivial kernel and $3-3$ entry of $Q$ is nonzero for $\tau \gg 0$. A similar proof of condition (94) to the one we just gave for crystal optics applies in cubic elasticity.

We need to check also that the cofactor systems associated to these equations are well posed. We will show this for the case where $Q_{L L}^{(i)}$ attains in Maxwell's equations, with a similar argument holding for $Q_{L L}^{(i i)}$. Consider the Maxwell system for $\epsilon$ replaced $\epsilon\left(-x_{1}, x^{\prime}\right)$, which is well posed. Make a change of variable $x_{1} \mapsto-x_{1}$. This returns $\epsilon$ to its original value and replaces $\xi_{1}$ by $-\xi_{1}$. Negating the functions $b, c$ then results in $\tilde{s}$ being negated and leaves $\tilde{p}, \tilde{r}$ unchanged. Next we pre and post multiply by $H^{T}$ and $H$ where

$$
H=\left[\begin{array}{lll}
0 & 1 & 0 \\
1 & 0 & 0 \\
0 & 0 & 1
\end{array}\right]
$$

to arrive at the desired cofactor system, which is now automatically well posed.

Once again the cofactor system for cubic elasticity can be seen to be well posed by a very similar argument.

\section{$5 \quad$ Propagation of singularities}

To understand how singularities of solutions to $(6)_{+}$propagate (c.f. [4]), we have seen that it is sufficient to understand propagation of singularities of the 
differential operator

$$
P_{s c}:=\partial_{t}^{2}-2 \partial_{\theta} \partial_{y_{2}}
$$

It is easy to compute the characteristics of this simple characteristic operator. If we then transform these characteristics back via the singular canonical transformation $\mathcal{T}$, we find that singularities are propagated along curves of the form

$$
\begin{aligned}
& t(s)=-\tau^{0} s \\
& \tau(s)=\tau^{0} \\
& x_{1}(s)=\sqrt{\alpha / \beta} \sin (2 \beta s) \\
& x_{2}(s)=2 \alpha s-(\alpha / 2 \beta) \sin (2 \beta s) \\
& x_{i}(s)=x_{i}^{0}, \quad 3 \leq i \leq n \\
& \xi_{1}(s)=\xi_{1}^{0} \cos (2 \beta s) \\
& \xi_{i}(s)=\xi_{i}^{0}, \quad 2 \leq i \leq n
\end{aligned}
$$

where $\alpha$ is zero exactly at a double characteristic point, $\alpha \ll \beta$ and $x_{i}^{0}, \xi_{i}^{0}$ are constants. Also, $\tau^{0}$ must be such that the above curve lies in $\operatorname{Char}\left(P^{+}\right)$.

We can obtain a qualitative picture of the propagation of singularities near the double characteristic points by examining (102) for $\alpha \ll \beta$. In the case of $(6)_{+}$, we observe a narrowly winding helix (with $x_{2}$ almost proportional to the parametrization of (102), and $x_{1}, \xi_{1}$ oscillating with small amplitude). The qualitative propagation of singularities described here agrees with the description in [4] and ([3], fig. 4).

Finally, since our analysis is microlocal, and we have not examined the global propagation of singularities.

\section{References}

[1] J. L. Antoniano and G.A. Uhlmann, "A functional calculus for a class of pseudodifferential operators with singular symbols", Proc. Symp. Pure Math. $43,5-16$ (1985).

[2] M. Born and E. Wolf, "Principles of Optics", Pergamon Press, (1959).

[3] R. Burridge, "The singularity on the plane lids of the wave surface of elastic media with cubic symmetry", Quart. Journ. Mech. and Applied Math., 20(1), 41-56, (1967). 
[4] P.J. Braam and J.J. Duistermaat, "Normal forms of real symmetric systems with multiplicity", Indag. Math. (N.S.) 4(4), 407-421 (1993).

[5] Y. Colin de Verdiere, "The level crossing problem in semi-classical analysis I. The symmetric case", Annales de Institut Fourier 53, 1023-1054 (2003).

[6] Y. Colin de Verdiere, "The level crossing problem in semi-classical analysis II. The Hermitian case", Annales de Institut Fourier 54, 1423-1441 (2004).

[7] R. Courant and D. Hilbert, "Methods of Mathematical Physics, II", Wiley Classics, New York (1989).

[8] N. Dencker, "On the propagation of polarization in conical refraction", Duke Math. J. 57, 85-134, (1988).

[9] J. Duistermaat, "Fourier integral operators", Birkhäuser, Progress in Mathematics, (1996).

[10] J. Duistermaat and L. Hörmander, "Fourier Integral Operators II", Acta Mathematica 128, 183-269 (1972).

[11] A. Greenleaf and G. Uhlmann "Estimates for singular Radon transforms and pseudodifferential operators with singular symbols", Funct. Anal. 89(1), 202-232 (1990).

[12] V. Guillemin and G. Uhlmann, "Oscillatory integrals with singular symbols", Duke Math. J., 48, 251-267 (1981).

[13] V. Ivrii, "On wave fronts of solutions of the system of crystal optics", Soviet Math. Dokl. 20, 139-141 (1977).

[14] V. Ivrii "Wave fronts of solutions of symmetric pseudodifferential systems", Siberian Math. J. 20, 557-578 (1979).

[15] R. Melrose, "The wave equation for a hypoelliptic operator with symplectic characteristics of codimension two", J. Analyse Math. 44, 134-182 (1985).

[16] R. Melrose and G. Uhlmann, "Microlocal structure of involutive conical refraction", Duke Math. J. 46, 571-582 (1979).

[17] C.J. Nolan, "Permittivity recovery from multiple chararcteristic waves", SIAM J. App. Math 62, 448-461 (2001).

[18] C.J. Nolan and G. Uhlmann, "Geometrical optics for systems with multiplicity", Contemp. Math. 333(2003), 177-185.

[19] M. Taylor, "Pseudodifferential Operators", Princeton University Press, Princeton, New Jersey, (1981).

[20] F. Treves, "Introduction to pseudodifferential and Fourier integral operators", Volumes 1 and 2, Plenum Press, (1980).

[21] G. Uhlmann, "Light intensity distribution in conical refraction", Comm. Pure and App. Math. 35, 69-80, (1982). 